\title{
EXPERIMENTAL DESIGN FOR SOIL GAS RADON MONITORING
}

\author{
D. M. THOMAS," J. M. COTTER," D. HOLFORD”"
}

\begin{abstract}
-Hawaii Institute of Geophysics, 2525 Correa Rd, Honolulu, HI 96822 (USA)
"Pacific Northwest Laboratory, P.O. Box 999, Richland, WA 99352 (USA)
\end{abstract}

(Received December 13, 1991)

An effort to define and characterize the environmental effects that control the release and mobility of radon in the environment is presented. The results of our preliminary field experiments on the long-term study of our radon activity measurements are reported.

\section{Introduction}

Radon, the radioactive daughter product of the decay of radium, has been the subject of scientific inquiry since its initial discovery in 1900 by DORN. ${ }^{1}$ Because it is ubiquitous in the environment, it has been studied as a means to better understand such diverse processes as earthquake precursors ${ }^{2,3}$ atmospheric circulation patterns ${ }^{4}$, eruptive and non-eruptive volcanic processes ${ }^{5}$, and groundwater transport and mixing ${ }^{6}$ Field surveys of soil gas radon activities have also been widely used for mineral prospecting ${ }^{7}$ and geothermal resources assessment $\cdot 8,9$ The recent discovery that radon activities within dwellings can achieve levels that pose a significant risk of lung cancer has prompted renewed interest in the mechanisms of radon release and transport in the natural environment, within confines of human habitations, and across the interface separating the two 10,11

As a first step toward addressing the question of how radon is transported from the soil into human habitations, the present study was initiated in an effort to define and characterize the environmental effects that control the release and mobility of radon in the natural environment. our ultimate objective is to determine the relationship between soil gas radon activities and meteorological variables such as barometric pressure, rainfall, and wind speed, and to develop an accurate numerical model of the response function of radon to these variables. The present paper will report on the 
results of our preliminary field experiments and describe the monitoring protocol developed for a long-term study that is currently underway.

\section{Experimental Methods}

In order for us to define the relationship between radon transport in the soil and the meteorological influences that control radon mobility, our experimental design was required to meet a number of constraints: the radon sampling interval must allow us to resolve changes in radon activity at time constants equivalent to those of the meteorological changes that control soil gas movement; the detection system must provide us with a continuous record of radon activities over at least one annual seasonal cycle; the monitoring protocol should not significantly disrupt the patterns of radon movement or soil gas transport within the soil in response to natural environmental variables.

These constraints severely restricted the types of equipment that could be used for the acquisition of radon activity data. Sampling and analysis of soil gas radon activities using an automated scintillation cell apparatus was believed likely to disrupt normal soil gas flow patterns and would have provided only discrete measurements of soil gas radon activity. Dosimetry techniques were similarly unacceptable, either because they were inappropriate for field deployment or they required integration times that were much longer than the time constants of the environmental variations of interest. 12 The monitoring equipment that was found to best meet the requirements of our experiment consisted of battery powered, field portable, "passive" alpha particle detectors. The instruments, (Model 611 AlphaLogger, manufactured by Alphanuclear Corporation of Mississauga, Ontario) consist of a stainless steel housing containing a $400 \mathrm{~mm}^{2}$ silicon diffused junction detector protected behind an aluminized mylar window (Fig. 1). Within the housing are contained pulse conditioning and counting electronics, a non-volatile data buffer, and a micro-processor that controls both the counting interval and data downloading to surface equipment. External power is supplied from an $18 \mathrm{~V}$ battery pack via a four conductor external line that also serves as the data transfer line. The diffused junction detector is recessed to a depth of approximately $5 \mathrm{~cm}$ into the lower 
end of the housing; the short penetration depth of alpha particles in air thus limits the access of alphas to those that have been generated in the gas phase within the "sensitive volume" immediately below the detector surface. Although beta and gamma emissions generated by soil radionuclides can traverse the air gap between the soil and the detector, their ionization efficiency is much lower than that for alpha particles and, hence, pulses generated in the detector are much smaller and are filtered out in the counting circuitry.

Although not part of the basic instrument design, in our field use the detection volume is further isolated from the soil by a thin

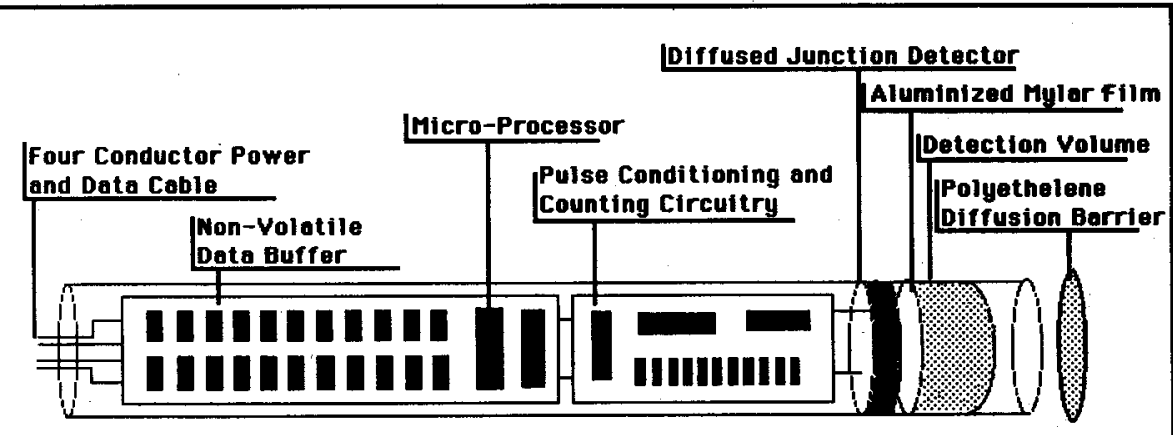

Fig. 1. Diagram of the Model 611 AlphaLogger showing detection volume, mylar window and diffused junction detector surface.

polyethylene film that serves as a diffusion barrier that excludes the entry of the very short-lived $\left(t_{1 / 2}=54 \mathrm{sec}\right) \mathrm{Rn}^{220}$ isotope. The rationale for excluding this isotope is that its lifetime is so short that it is unlikely to show a significant concentration response to the much longer term environmental effects that are the objective of this investigation.

The operation of the instrument is based on its ability to continuously detect and count alpha particle decays within the detection volume over a preset 15 minute interval, record the number of counts in a non-volatile memory buffer, and then continue counting the succeeding 15 minute interval. A continuous record of 15 minute interval counts is stored in the data buffer for counting durations of up to 40 days before the initial data are overwritten. Retrieval of data from the internal buffer can be accomplished by directly 


\section{M. THOMAS et al.: EXPERIMENTAL DESIGN FOR SOIL GAS RADON}

linking the instruments to a laptop computer through its RS-232 communications port or by downloading through a modem. The passive nature of the radon detection allows us to monitor radon activities in the soil column for extended periods of time with essentially no disturbance to the soil or soil gas transport processes associated with monitoring or periodic downloading of data.

Prior to installation of the instruments in the field, the alpha particle counters were calibrated in the laboratory using an enclosed chamber containing soil from the field site. This was accomplished by sealing the instrument into the exposure chamber and, during the period of equilibration of the "head gas" within the chamber, samples of gas were withdrawn and analyzed in a silver activated zinc sulfide scintillation apparatus that had been calibrated using a radium standard reference sample obtained from the U.S. National Institute of Standards and Technology. The calibration thus obtained allowed us to convert alpha particle count rates to radon activities in the soil gas.

In addition to radon activity measurements, we also maintained a meteorological and soil physics monitoring program to enable us to correlate changes in environmęntal conditions with changes in radon activity. Barometric pressure was monitored using a Honneywell $140 \mathrm{PC}$ series pressure gauge attached to a Hydronet Model 11 analog data recorder. Differential gas pressures between the soil surface and the depths of the radon instruments were monitored using Honeywell 140PC differential pressure gauges and Model 11 data recorders. During the initial phase of the experiment, rainfall data were obtained using a manually recorded rain gauge. However, during the latter part of the investigation, a sierra Misco Tipping Bucket rain gauge was employed for this purpose. Soil moisture levels were monitored using ceramic disk detectors supplied by Aguatronics but, due to failure of these devices, during the course of the experiment, porous cup tensiometers were employed for spot measurement of soil water tension. Wind speed and direction were monitored using a R.M. Young wind Sentry attached to the Hydronet Model 15 frequency logger.

At the outset of the experiment, we conducted a preliminary test. of our monitoring equipment to determine whether the radon activities were sufficient to provide adequate counting statistics within an integration interval that would be consistent with the environmental effects of interest. In this test, a single instrument was installed at the proposed test site as shown in Figure $2 \mathrm{a}$; a hole was augered 


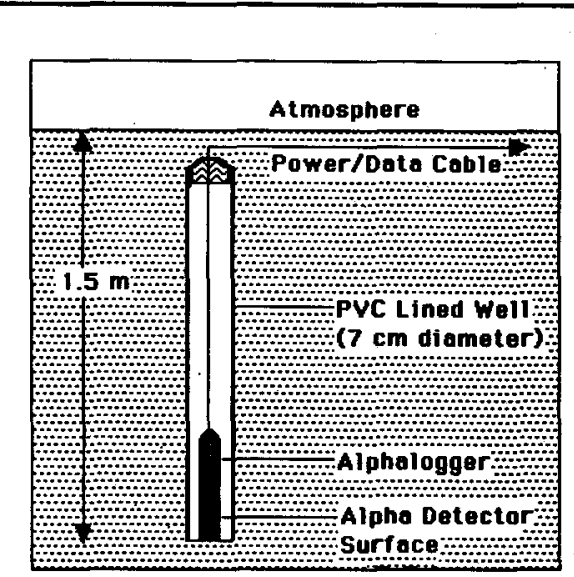

Figure 2a

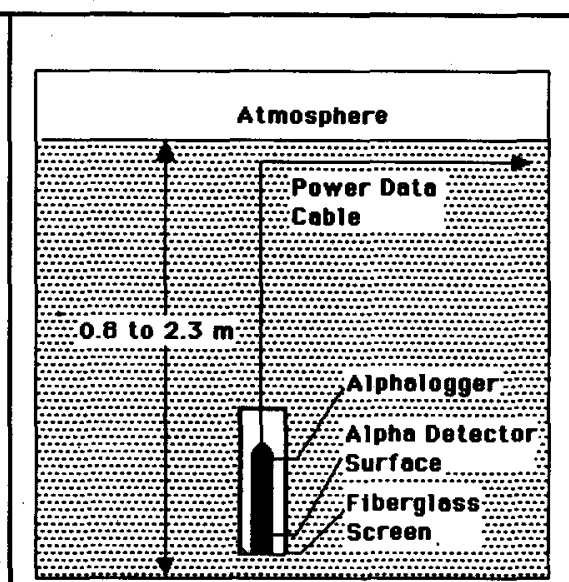

Figure 2b

Fig. 2. a. Diagram of initial field deployment protocol for the radon monitoring instruments.

b. Diagram of revised deployment protocol.

into the soil column to a depth of $1.5 \mathrm{~m}$ and lined with a plastic sleeve to a point near the soil surface; the radon instrument was placed at the bottom of the well (with the detection surface facing downward); the power/data cable extended to the surface through a solid plug that was used to seal the top of the instrument well; and finally the top of the unlined surface portion of the well was back-filled with soil. Monitoring of radon activities at this station continued for approximately ninety days prior to retrieval of the radon instrument.

\section{Results and Discussion}

The radon activity data obtained during the preliminary monitoring interval, presented in Figures 3 and 4 , show that the radon count rates at $1.5 \mathrm{~m}$ depth in the soil ranged from $400 \mathrm{c} / \mathrm{hr}$ to $500 \mathrm{c} / \mathrm{hr}$ corresponding to activities of approximately 20 to $30 \mathrm{~Bq} / \mathrm{l}$ of soil gas. The count rates, although substantial, were not high enough to justify using the minimum integration times of 15 minutes, but require smoothing over periods of from one to twelve hours to 
D.M. THOMAS et al.: EXPERIMENTAL DESIGN FOR SOIL GAS RADON

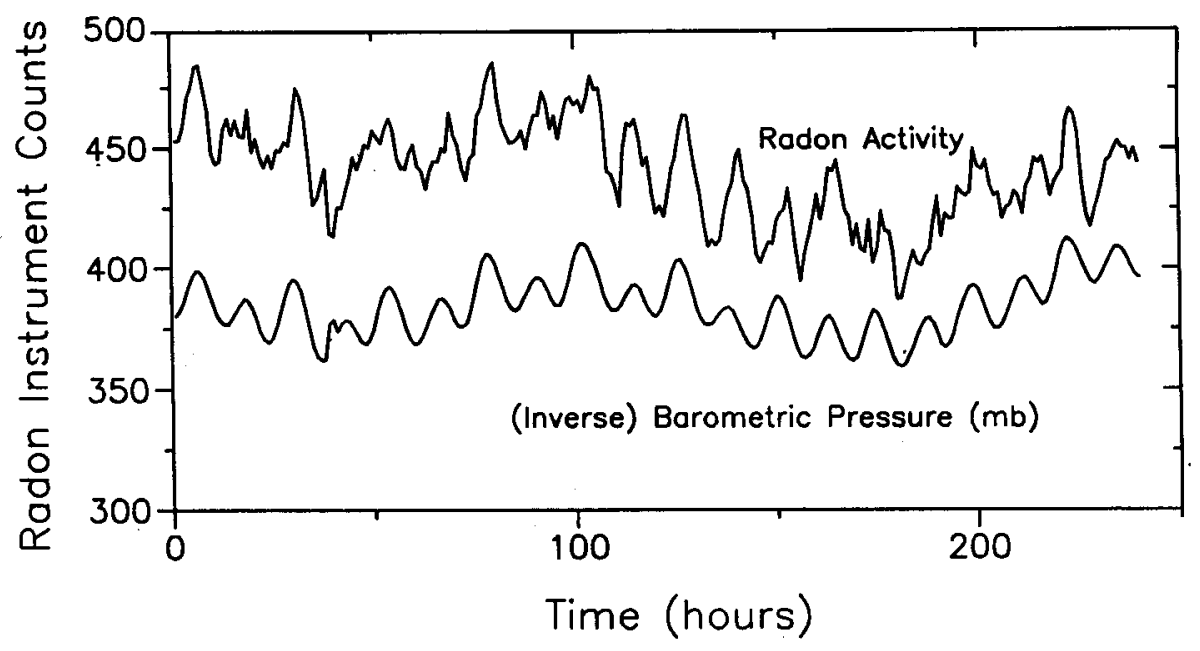

Fig. 3. Plot of radon hourly count rates observed at $1.5 \mathrm{~m}$ depth in soil and the inverse barometric pressure (given in millibars $x$ 10).

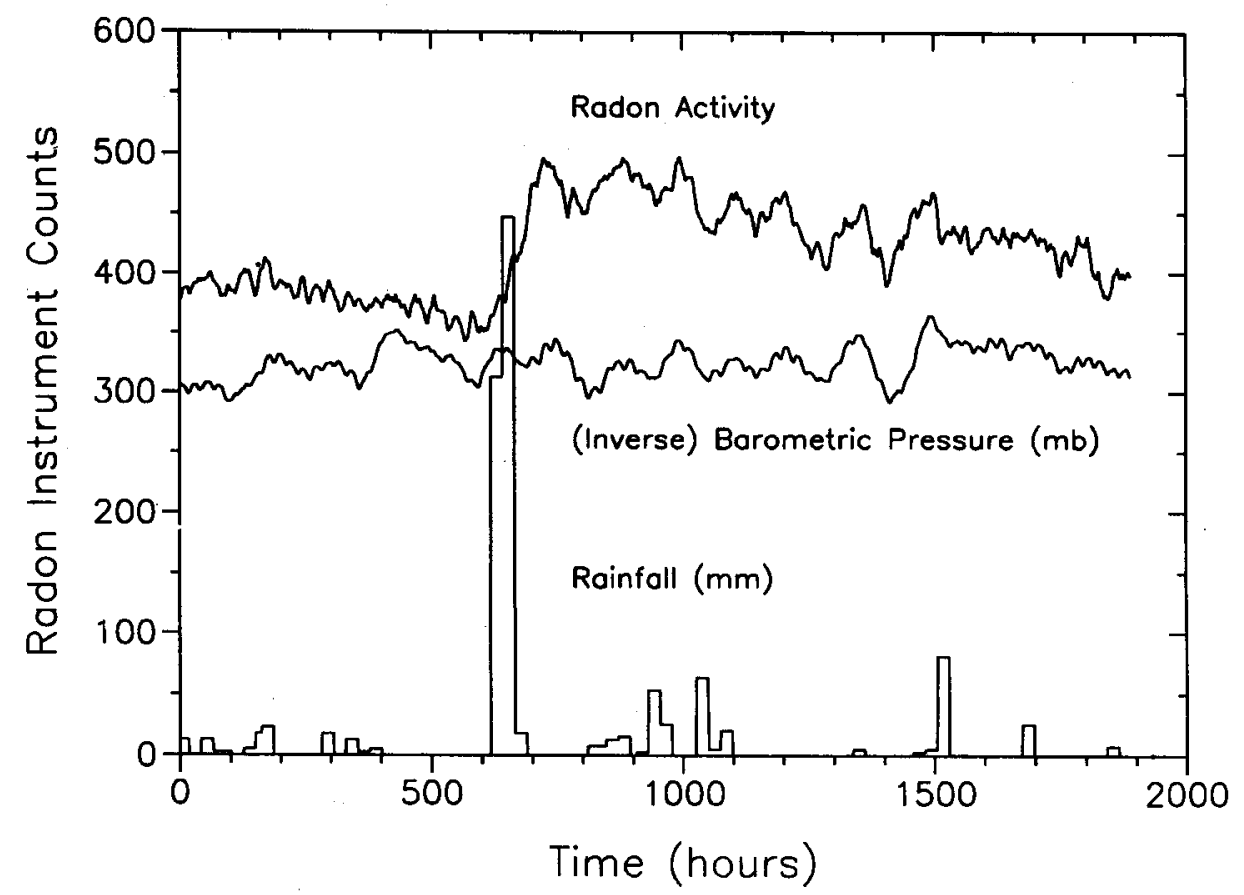

Fig. 4. Plot of radon hourly count rates at $1.5 \mathrm{~m}$ depth along with inverse barometric pressure (in millibars $x$ 10) and rainfall (in millimeters). 
reduce the random variability in the counting rates. The data also show that soil gas radon concentrations undergo significant variations over time intervals that range from hours to weeks. comparison of radon activity data, smoothed over a four hour moving window, with barometric pressure (Fig. 3) shows a clear inverse correlation with the short-term variation in atmospheric pressure known as the s-2 pressure signal. The latter is the result of solar tidal effects on the atmosphere and has a 12 hour period ${ }^{13}$; harmonic analysis of an extended sample of radon activities has confirmed the presence of a 12 hour signal within the longer term fluctuations in radon activity.

When the data are smoothed over a 12 hour interval, longer term and somewhat larger variations become apparent (Fig. 4). These are also correlated with changes in barometric pressure but, in this case, result from synoptic pressure variations associated with the passage of storm systems across the island chain. The latter pressure variations appear to generate changes in radon activities that range up to approximately $15 \%$ of the total activity observed at the $1.5 \mathrm{~m}$ depth of the instrument.

These results are consistent with earlier investigations of soil gas radon activities that have shown that synoptic barometric pressure changes are capable of displacing the radon concentration profile downward or upward in response to, respectively, rising or falling barometric pressure: $14,15,16$ Although the short term radon variations have not been generally recognized before, it is clear that the semi-diurnal variations in the barometric pressure signal associated with the s-2 pressure wave result from the same effect.

The largest change in radon activity, however, is clearly associated with a rainfall event (hour 620 in Figure 4) that has substantially increased the soil gas radon concentrations. This response may be the result of two possible phenomena. The water influx may displace pore-bound radon from the soil micro-pores into the meso- or macro-pore volume within the soil where it is detected by the instruments. Alternatively, hydration of the soil may reduce its permeability and restrict advective and diffusive exchange of soil gas with atmospheric air. We believe that the latter mechanism is the more likely, however, analysis of long-term radon response data will be required in order to confirm this hypothesis.

A more subtle effect is also present in the radon response to the rainfall event: the relative magnitude of the post-rainfall radon variations induced by the diurnal barometric pressure signal 


\section{M. THOMAS et al.: EXPERIMENTAL DESIGN FOR SOIL GAS RADON}

are significantly larger than those that occurred prior to the rainfall and show a much stronger correlation with the pressure signal. This suggests that transport of soil gas, and its exchange with radon poor atmospheric air, is more effective under wet soil conditions than with dry soil. However, this is clearly contrary to the observed increase in post-rainfall radon concentration that results from a moisture-induced decrease in soil gas permeability and, hence, some other process may be interfering with the pressure driven soil gas transport. We believe that the process responsible for these observations is preferential soil gas flow along and around the lined probe well that enhances exchange of radon-deficient air with the soil gas. Under dry soil conditions, the difference in permeability between the probe well and the soil matrix is small and air exchange through the soil is only slightly less effective than that through the probe well. As the soil hydrates and its permeability decreases, the barometric pressure driven air exchange through the probe well increases, generating the larger semi-diurnal variations in soil gas radon activity.

These observations are significant both for our experimental design as well as in terms of the longer range objectives of our research program. With respect to our monitoring protocol, it is clear that installation of the monitoring instruments must minimize any potential for preferential exchavge of radon rich soil gas with atmospheric air. In order to meet uis requirement, the field installation of the radon instruments for our long-term study has omitted the lined probe well and has, instead, housed the detector in a plastic housing that is buried to the depth of interest with the only continuous connection between the instrument and the surface consisting of the sealed power/data cable (Fig. 2b).

The long-term monitoring study now underway, has installed two separate arrays of radon instruments at the field site with each array consisting of a radon instrument located at a depth of $0.8 \mathrm{~m}$, $1.3 \mathrm{~m}$, and $2.3 \mathrm{~m}$. Monitoring at three depths will allow us to define the depth range over which the pressure and moisture effects are important and will enable us to better separate the physical processes that affect radon transport. The use of identical test/control radon arrays will also enable us to artificially change soil conditions (e.g. moisture, permeability, etc.) at the test array while monitoring the control. Using this approach, we will be able to isolate and observe the individual effects of changes in soil 
conditions and to numerically model their response functions independently. The independently verified numerical models can then be used to construct an integrated model of radon response to environmental variables.

In an effort to better monitor soil conditions, soil moisture and temperature probes were installed adjacent to the radon instruments at each of the three depths and differential pressure monitoring tubing at the $0.8 \mathrm{~m}$ and $2.3 \mathrm{~m}$ depths.

Although monitoring at the field station is still underway, our initial results have indicated that our modification of the radon deployment protocol has eliminated the preferential advection of soil gas around the monitoring probe. The monitoring results have also tended to confirm the initial findings that rainfall induced changes in soil moisture have the greatest impact on subsurface radon concentrations but that synoptic and diurnal barometric pressure variations also exert a strong control over soil gas advection within the shallow soil column (Fig. 5). Analysis and modelling of these results are currently underway.

\section{Conclusions}

The results of our preliminary investigation of radon activities in the shallow soil column have shown that both diurnal and synoptic barometric pressure changes can significantly affect the exchange of atmospheric air with soil gas and that rainfall induced changes in soil moisture and soil permeability can substantially affect rates of soil gas transport in the shallow soil column. Our results have also shown, however, that even minor changes in soil permeability induced by experimental protocols can substantially affect soil gas transport processes and observed radon activities.

A broader, and possibly more significant, application of the latter result is that other man-made pathways through the shallow soil layer can act as a preferred pathway for the transport of radon-rich soil gas from depth in the geologic column. Hence, the presence of a basement beneath a dwelling, and its mode of construction, may have a far higher impact on the extent of radon exposure experienced by the residents of a dwelling than does the inherent radon activity of the surrounding soils. 
D. M. THOMAS et al.: EXPERIMENTAL DESIGN FOR SOIL GAS RADON

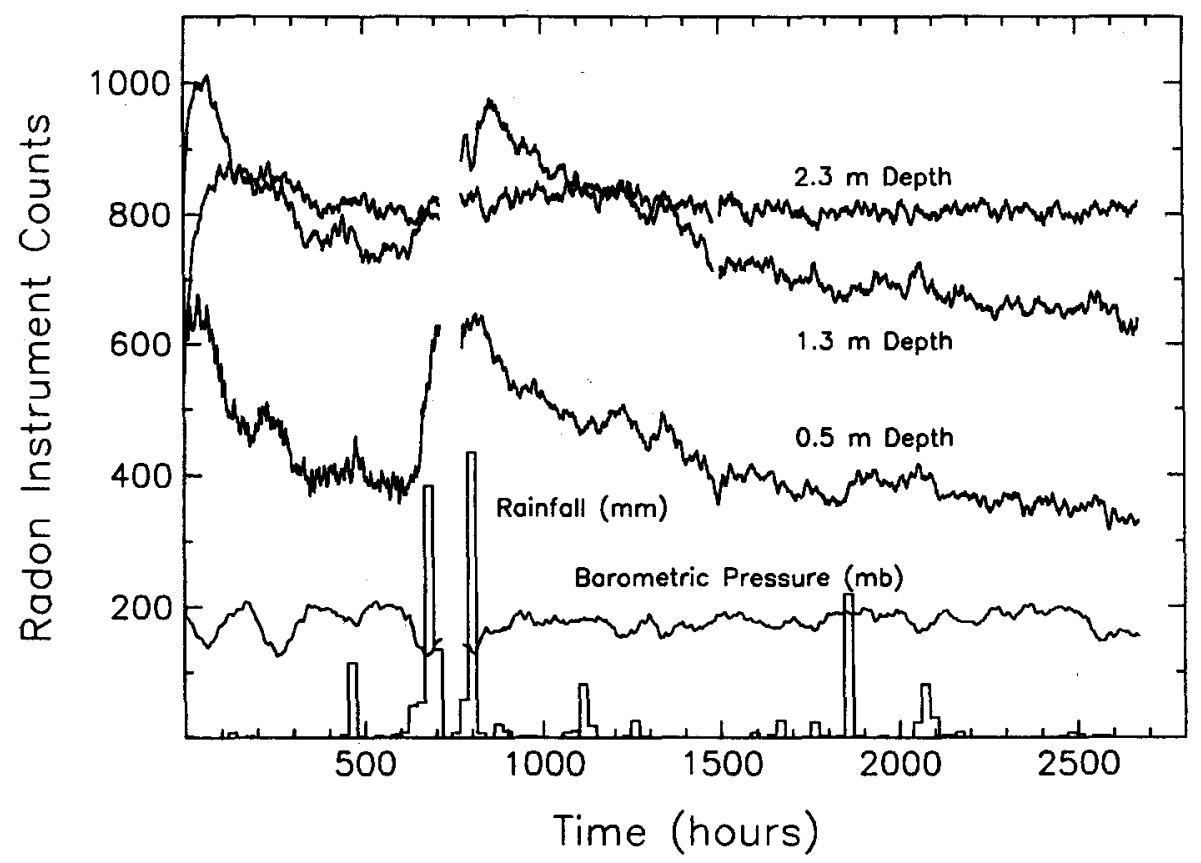

Fig. 5. Plot of radon hourly count rates at three depths in the soil column. Also shown are daily rainfall (in millimeters) and $\Delta \mathrm{mb} X 10$ ( $\Delta \mathrm{mb}$ calculated as [B.P. X 1000]-1000)

\section{Acknowledgment}

This work is being supported by the U.S. DOE Office of Health and Environmental Research Contract No. DE-FG03-88ER6063. The authors would like to acknowledge the assistance and encouragement of Drs. Curtis Olsen, Michael Reimer, and w. Forster in the planning and execution of this investigation. We would also like to acknowledge the Alphanuclear corporation for their cooperation and assistance in making design modifications of their instrumentation to enhance its compatibility with our experimental objectives. This is school of Ocean and Earth Science and Technology Contribution No. 2687.

\section{References}

1. W. GERRARD, Gas Solubilities, Pergammon Press, Oxford, 1980, p. 162.

2. E. HAUKSSON, J. Geophys. Res., 86(B10) (1981) 9397. 
3. D. THOMAS, PAGEOPH, 126(2-4) (1988) 241.

4. M. WILKENING, Proc. Conf. on the Natural Radiation Environment, 1981, p. 565.

5. D. THOMAS, Proc. Conf. on Radon Monitoring in Radiation Protection, Environmental Radioactivity, and Earth Sciences, Trieste, 1990, p. 295.

6. H. A. WOLLENBERG et al., PAGEOPH, 122 (1984/1985) 327.

7. J. E. GINGIRICH, Trans. Soc. Min. Eng., 258 (1975) 61.

8. L. SEMPRINI, P. KRUGER, Geothermics, 13 (1984) 103.

9. M. COX, Geophys. Res. Lett., 7(4) (1980) 283.

10. A. V. NERO, LBL Research Rev. 11(4) (1986) 15.

11. A. V. NERO, W. W. NAZAROFF, Rad. Prot. Dos., 7(1-4) (1985) 23.

12. D. THOMAS, K. CUFF, M. COX, J. Geophys. Res., 91(B12) (1986) 12186.

13. S. CHAPMAN, Compendium of Meteorology, American Meteorological Society, 1951, p. 510.

14. A. TANNER, Natural Radiation Environment III, 1980, p. 5.

15. S. D. SCHERY, D. GAEDERT, M. WILKINING, J. Geophys. Res., 87(B4) (1982) 2969.

16. S. D. SCHERY, D. GAEDERT, M. WILKINING, J. Geophys. Res., 89(D5) (1984) 7299. 